\title{
Aves silvestres infestadas por Phthiraptera (Insecta) na Zona da Mata Norte de Pernambuco, Brasil
}

\author{
Sônia Aline Roda ${ }^{1}$ \\ Ângela Maria Isidro de Farias ${ }^{1}$
}

\begin{abstract}
Wild birds infested by Phthiraptera (Insecta) in Pernambuco North Zona da Mata. Studies were carried out on the relationship between Phthiraptera and wild birds captured by mist nets at the Engenho Independência, at Vicência's county. Three families of Phthiraptera were identified. The Menoponidae were the one with the highest number of hosts. They were specially found in the dorsal face of the remiges. The Trochiloecetidae were only seen infesting the neck of birds belonging to the hummingbirds. The Philopteridae were observed on birds from three orders showing the highest variety of distribution on the birds.

KEY WORDS. Phthiraptera, ectoparasites, wild birds, Pernambuco
\end{abstract}

Os phthirápteros são bem adaptados ao ectoparasitismo e todos são parasitos obrigatórios de aves e mamíferos (CHENG 1964; Noble \& Noble 1965). Neles, todos os estádios de desenvolvimento do ciclo biológico são completados sobre o corpo de um mesmo hospedeiro (CHANDLER 1955; BAER 1971; ASKEW 1973; MARSHALl 1981; BARKER et al. 1991). Os ovos, ninfas e adultos dependem totalmente da proteção das penas e pêlos de seus hospedeiros contra a dessecação (BARKER 1994), além da alimentação que o hospedeiro lhes proporciona.

Como ectoparasitos permanentes, os phthirápteros tendem a ser altamente específicos em relação ao hospedeiro (MARSHALL 1981) e os contatos entre indivíduos da mesma espécie, seja dos pais com os filhos ou o contato sexual, asseguram a manutenção entre os hospedeiros de uma mesma espécie (MARSHALL 1981; RòzSA 1993).

No Brasil, os phthirápteros de aves tem sido pouco estudados, não se sabendo com certeza, se atuariam como vetores potenciais de doenças, bem como quais são os danos reais que poderiam causar em seus hospedeiros. Devido a esta carência de informações, o presente trabalho teve como objetivo, identificar os phthirápteros coletados, determinar a infestação desses ectoparasitos em seus respectivos hospedeiros, além de relacioná-los aos sítios preferenciais de parasitismo.

\section{MATERIAL E MÉTODOS}

As aves foram capturadas no Engenho Independência ( $35^{\circ} 26^{\prime} \mathrm{W}$ e $\left.7^{\circ} 39^{\prime} \mathrm{S}\right)$, situado no município de Vicência, Zona da Mata Norte de Pernambuco, em quatro

1) Departamento de Zoologia Centro de Ciências Biológicas, Universidade Federal de Pernambuco. Avenida Professor Morais Rego 1235, Cidade Universitária, 50670-420 Recife, Pernambuco, Brasil.

E-mail: saroda@hotlink.com.br 
períodos: junho, setembro e dezembro de 1995 e março de 1996. Foram utilizadas redes de neblina, distribuídas nos diferentes ambientes da área de estudo (pomar, jardim, pasto, margens de rio e açudes e capoeirinha).

As aves foram examinadas cuidadosamente em relação à presença de ectoparasitos, utilizando-se uma fonte de luz dirigida à plumagem, sobre um papel branco, assim facilitando a visualização e coleta de possiveis ectoparasitos que, eventualmente, caíssem fora do corpo da ave durante a manipulação. A plumagem do corpo era afastada com auxílio de pincel (seco ou umedecido em água), manualmente ou por sopro. As rêmiges e retrizes eram observadas contra a luz, a fim de facilitar a visualização dos ectoparasitos.

Os ectoparasitos visíveis foram retirados do hospedeiro com auxílio de pinça ou pincel fino, juntamente com as penas (no caso das rêmiges e retrizes) ou removendo-se a pena (no caso da cabeça e corpo). As amostras foram transferidas para frascos de vidro devidamente individualizados e numerados de acordo com a ficha de campo da ave hospedeira e separados por região do corpo da ave, e em seguida fixadas em álcool a $70^{\circ}$.

No Laboratório de Invertebrados Terrestres do Departamento de Zoologia da Universidade Federal de Pernambuco procedeu-se à preparação, montagem entre lâmina e lamínula e identificação. Alguns exemplares preservados em álcool a $70^{\circ}$ foram posteriormente enviados a especialistas.

A infestação por phthirápteros para cada espécie de ave hospedeira foi quantificada usando-se a intensidade média e a prevalência, segundo MARGOLIS et al. (1982). Nas aves, os sítios ectoparasitados foram analisadas através da análise de variância, segundo o método de Kruskall-Wallis, e as médias pela comparação múltipla de Kruskall-Wallis, ou pelo teste t de Student. As análises estatísticas foram realizados a um nível de significância de 5\%.

\section{RESULTADOS}

Do total de 160 aves examinadas, apenas 28 indivíduos (17,5\%) de 15 espécies (Tab. I) estiveram infestadas por phthirápteros pertencentes às subordens Amblycera (Trochiloecetidae e Menoponidae) e Ischnocera (Philopteridae).

Da família Trochiloecetidae, o gênero Trochiloecetes, foi observado em Glaucis hirsuta e Eupetomena macroura (Trochilidae); o gênero Trochiliphagus, apenas em E. macroura.

Tanto em G. hirsuta, como em E. macroura, os adultos de Trochiloecetes foram encontrados na região ântero-inferior e face dorsal do pescoço. Posicionavam-se com a face anterior do corpo direcionada para baixo e com as garras presas no cálamo. Os ovos e ninfas das espécies deste gênero apresentavam-se fixos nas bases das penas, nos mesmos locais onde também foram encontrados os adultos.

Verificou-se, para estes dois gêneros de phthirápteros, apenas a presença de um indivíduo adulto ou ovo por pena. Nenhum phthiráptero desta família foi observado locomovendo-se sobre os hospedeiros. A prevalência e a intensidade média são indicadas na tabela I. 
Tabela I. Prevalência, intensidade média e sítios preferenciais de infestação das familias Trochiloecetidae, Menoponidae e Philopteridae (Phthiraptera, Insecta) em aves silvestres capturadas no Engenho Independência, Vicência, Pernambuco. (n) Número de indivíduos capturados, (N) número de indivíduos infestados, (PT) número de phthirápteros, (NI) não identificados, (NQ) não quantificados, (FD) face dorsal, (FV) face ventral, $(R M)$ rêmiges, $(R T)$ retrizes, $(P)$ papo, $(P E)$ pescoço, $(N U)$ nuca, $(A)$ abdômen, $(V)$ ventre, $(P O)$ regiäo peri-ocular.

Hospedeiros $\quad n \quad N$ PT Trochiloecetidae Menoponidae Philopteridae $\begin{gathered}\text { Prevalência Intensidade } \\ \text { média }\end{gathered}$ Sitios ectoparasitados

\begin{tabular}{|c|c|c|c|c|c|c|c|c|c|}
\hline \multicolumn{10}{|l|}{ Jacanidae } \\
\hline Jacana jacana (Linneus, 1758) & 4 & 2 & 3 & - & NI & - & 50,0 & 1,5 & FD RM, P \\
\hline \multicolumn{10}{|l|}{ Columbidae } \\
\hline Columbina minuta (Linneus, 1766) & 8 & 2 & NQ & - & - & Columbicola passerinae & 25,0 & NQ & FV e RD RM e RT \\
\hline Columbina talpacoti (Temmink, 1811) & 5 & 2 & NQ & - & - & Columbicola passerinae & 40,0 & NQ & FV e FD RT \\
\hline \multicolumn{10}{|l|}{ Trochilidae } \\
\hline Glaucis hirsuta (Gmelin, 1788) & 15 & 5 & 15 & Trochiloecetes & - & - & 33,3 & 3,0 & PE \\
\hline \multirow[t]{2}{*}{ Eupetomena macroura (Gmelin, 1788) } & 37 & 4 & 4 & Trochiloecetes & - & - & 10,8 & 1,0 & PE \\
\hline & & 1 & 2 & Trochiliphagus & - & - & 2,7 & 2,0 & $P E$ \\
\hline \multicolumn{10}{|l|}{ Alcedinidae } \\
\hline Chloroceryle americana (Gmelin, 1788) & 1 & 1 & 1 & - & - & Quadraceps & 100,0 & 1,0 & FD RT \\
\hline \multicolumn{10}{|l|}{ Furnariidae } \\
\hline Certhiaxis cinnamomea (Cory, 1916) & 7 & 1 & 7 & - & - & Stumidoecus & 14,3 & 7,0 & NU \\
\hline \multicolumn{10}{|l|}{ Tyrannidae } \\
\hline Tolmomyias sulphurescens (Hartert \& Goodson, 1917) & 1 & 1 & 1 & - & Myrsidea & - & 100,0 & 1,0 & A \\
\hline Tyrannus melancholicus (Lichtenstein, 1888) & 2 & 1 & 6 & - & - & Stumidoecus & 50,0 & 6,0 & $\mathrm{PO}, \mathrm{PE}$ \\
\hline \multicolumn{10}{|l|}{ Hirundinidae } \\
\hline Stelgidopteryx ruficollis (Vieillot, 1885) & 65 & 2 & 2 & - & Myrsidea & - & 3,0 & 1,0 & FD RM \\
\hline \multicolumn{10}{|l|}{ Troglodytidae } \\
\hline Troglodytes aedon Naumann, 1823 & 3 & 1 & 11 & - & - & $\mathrm{NI}$ & 33,3 & 11,0 & V \\
\hline \multicolumn{10}{|l|}{ Muscicapidae } \\
\hline Turdus rufiventris (Cory, 1916) & 2 & 2 & 2 & - & Myrsidea & - & 100,0 & 1,0 & FD RM \\
\hline \multicolumn{10}{|l|}{ Emberizidae } \\
\hline Thlypopsis sordida (Lefresnaye \& d'Orbigny, 1837) & 7 & 1 & 1 & - & Myrsidea & - & 14,3 & 1,0 & A \\
\hline Paroaria dominicana (Linneus, 1758) & 2 & 1 & 1 & - & NI & - & 50,0 & 1,0 & FD RM \\
\hline Molothrus badius (Spix, 1824) & 1 & 1 & 1 & - & $\mathrm{NI}$ & - & 100,0 & 1.0 & FD RM \\
\hline Total/Média & 160 & 28 & - & - & - & - & 45,4 & - & - \\
\hline
\end{tabular}


Os hospedeiros, prevalência, intensidade média e os sítios parasitados por Menoponidae são apresentados na tabela I. Verificou-se que os phthirápteros desta família ocorreram com maior freqüência na face dorsal das rêmiges, entretanto os resultados da análise estatística não mostraram diferenças estatísticas significativas $(\mathrm{t}=1,0 ; \mathrm{gl}=13 ; \mathrm{p}>0,05)$ entre os demais sítios parasitados.

Os menoponideos encontrados em Jacana jacana (Jacanidae) caminhavam muito lentamente, em contraposição ao gênero Myrsidea, observado em Thlypopsis sordida (Emberizidae) e Turdus rufiventris (Muscicapidae) com movimentos rápidos. Em Stelgidopteryx ruficollis (Hirundinidae) e Tolmomyias sulphurescens (Tyrannidae) não foram observados movimentos.

Os ovos de Menoponidae quando presentes em T. rufiventris apresentavamse agrupados sob a forma de "cachos", de cor amarela-pálida, envolvendo quase completamente as penas das regiões pós-ocular e auricular.

Relativamente aos Philopteridae, os hospedeiros, prevalência, intensidade média e sítios preferenciais de infestação são apresentados na tabela I. Nas retrizes foi encontrado um maior número de indivíduos, no entanto, os testes estatísticos não revelaram diferenças significativas (Kruskall-Wallis $(\mathrm{H} 1)=2,1636 ; \mathrm{p}>0,05$ ) entre os demais locais ectoparasitados.

Os indivíduos da espécie Columbicola passerinae (Philopteridae) localizavam-se paralelamente às barbas das penas, com a cabeça voltada em direção do raque. Embora permanecendo fixos no vexilo da pena, a aproximação da pinça no momento da coleta, fê-los apresentar movimentos rápidos em direção ao raque, paralelos às barbicelas, permitindo que vários indivíduos escapassem, impedindo desse modo a contagem desses indivíduos.

A espécie do gênero Quadraceps, foi coletada sobre Chloroceryle americana (Alcedinidae), e a do gênero Sturnidoecus, sobre Certhiaxis cinnamomea (Furnariidae) e Tyrannus melancholicus (Tyrannidae) (Tab. I).

Em C. cinnamomea e T. melancholicus os phthirápteros adultos do gênero Sturnidoecus posicionavam-se com a cabeça voltada para baixo, direcionada para o raque, na pena. Em C. cinnamomea foram observados ovos e córion presos no raque e no vexilo da face ventral (na porção penácea normal e porção plumácea) das penas da nuca, junto com indivíduos adultos. Em T. melancholicus os ovos foram encontrados presos no hiporraque, logo após o umbílico superior, também nas penas da nuca.

Foi observado um indivíduo muito jovem de Troglodytes aedon (Passeriformes, Troglodytidae) infestado por um número elevado de phthirápteros jovens da família Philopteridae e apresentando as penas da região ventral muito corroídas, provavelmente devido ao ataque desses ectoparasitos.

Os dados referentes a Ordem Passeriformes (famílias Furnariidae, Tyrannidae, Hirundinidae, Troglodytidae, Muscicapidae e Emberizidae) (Tab. I) revelaram que 11 indivíduos $(6,87 \%)$ pertencentes a nove espécies encontravam-se infestados por phthirápteros.

Em outras espécies de aves não relacionadas na tabela apresentada também foram coletados ovos (lêndeas) não identificados. Assim, em Chlorestes notatus 
Reichenbach, 1795 e Amazilia fimbriata (Gmelin, 1788) (Trochilidae) eles foram observados na face inferior do pescoço, enquanto que em Thraupis sayaca (Linnaeus, 1766) (Emberizidae) na região gular.

\section{DISCUSSÃO E CONCLUSÕES}

Segundo ONIKI (1995), a separação dos gêneros Trochiloecetes e Trochiliphagus pode ser feita através do comportamento dos indivíduos e pelos diferentes sítios de infestação no corpo do hospedeiro onde eles são encontrados. As espécies de Trochiloecetes põem seus ovos na parte dorsal do pescoço do hospedeiro, permanecendo perto deles, como observado em Ramphodon naevius (Dumont, 1818)(Apodiformes, Trochilidae) por ONIKI \& EMERSON (1982). Quando a infestação é alta, os adultos e os ovos ocorrem também nos lados e na região frontal do pescoço (ONIKI 1995).

No presente trabalho foram encontrados dois indivíduos adultos de Trochiliphagus juntamente com alguns ovos na região anterior e face dorsal do pescoço em E. macroura. Embora ONIKI (1995) considere que os indivíduos adultos não costumam permanecer perto dos seus ovos. Esta autora observou ainda que Trochiliphagus desloca-se rapidamente por entre a plumagem do corpo do hospedeiro.

Com relação ao número de espécimens, observou-se que Trochiloecetes sp. ocorreu em maior número que Trochiliphagus sp.. Dados semelhantes foram encontrados por CARRIKER (1960), CLAYTON et al. (1992) e ONIKI (1995).

Entre os Menoponidae, o único gênero identificado no presente trabalho foi Myrsidea, ocorrendo em quatro das sete espécies de aves parasitadas por esta família CLAYTON et al. (1992) encontraram-no infestando 25 espécies de aves, 20 das quais incluídas na ordem Passeriformes. Peters (1936), apud WHEELER \& THRELfall (1986), reportou M. incerta em 25 espécies de Passeriformes, e WHEELER \& THRElfall (1986), assinalaram três hospedeiros para esta espécie. Segundo ONIKI (1990), as espécies de Myrsidea são pouco estudadas em aves sul-americanas; mesmo assim, encontrou Myrsidea sp. em 10 indivíduos, pertencentes a sete famílias da ordem Passeriformes, entre 16 espécimes capturados na Amazonia.

Os movimentos do gênero Myrsidea observados em alguns dos seus hospedeiros pode ser um indicativo de que, quando adultos, este gênero não apresente um sítio preferencial de infestação.

Os ovos de phthirápteros, foram aqui reconhecidos no pescoço e na cabeça de aves das famílias Trochilidae, Furnariidae, Tyrannidae e Muscicapidae, enquanto que ONIKI \& WILLIS (1991) assinalaram-nos no mento, loros, acima dos olhos e no topo da cabeça. Acredita-se que nestes locais, os ovos não podem ser retirados pelas aves, no momento em que elas se coçam ou alisam suas penas.

As variações morfológicas apresentadas pelos phthirápteros, como por exemplo, a compressão do corpo, permitem e limitam a colonização de diferentes regiões do corpo em uma mesma ave. Geralmente, os phthirápteros apresentam a cutícula de cor marrom, entretanto, aqueles que infestam aves exibem uma coloração críptica, podendo alterá-la de acordo com o sítio onde se encontrem no hospedeiro (MARSHALl 1981). 
As adaptações comportamentais estão diretamente relacionadas com o sítio de infestação ocupado pelos phthirápteros, nos respectivos hospedeiros, assim instalando-se preferencialmente nos locais onde não possam ser predados pelos hospedeiros, ou que não ocorra muita atividade de auto-catação ("self-grooming") ou movimentação física (MARSHALL 1981) ou ainda onde as atividades de alisar com o bico ("preening") não sejam muito intensas (STOCK \& HUNT 1989).

Como o pescoço e a cabeça não são facilmente alcançados pela ave durante o alisar das penas, os phthirápteros desenvolveram fortes mandíbulas com o propósito de melhor se fixarem ao hospedeiro (CLAY 1949). Para BAER (1971), uma deformidade no bico de uma ave poderia lhe impedir de limpar a sua plumagem e, consequentemente, manter a infestação em caráter mais exarcebador que as demais. BOYD (1951) ao constatar que Sturnus vulgaris (Sturnidae) utilizava o bico para a retirada de ectoparasitos, verificou que um indivíduo com o bico deformado encontrava-se intensamente parasitado por phthirápteros. Condições similares foram observadas em aves silvestres por ASH (1960), FosTER (1969) e BAER (1971) e em aves de granja por KARTMAN (1950) e FIGUEIREDO et al. (1993).

A localização e posição de Columbicola passerinae nos hospedeiros infestados é semelhante à de C. columbae observada por STENRAM (1956), mantendo o corpo paralelo às barbas e a cabeça apontada como uma lança para o eixo longitudinal da pena, permitindo assim, um deslocamento rápido durante as atividades de "preening" por parte do hospedeiro.

Em Troglodytes aedon, infestado por muitos phthirápteros jovens da família Philopteridae, visto que nele não foi observado ovos, isto vem reforçar a afirmação de FosTER (1969), de que as aves jovens em geral não apresentam ovos em sua plumagem.

O percentual de aves infestadas por phthirápteros no presente trabalho $(17,5 \%)$, pode ser considerado muito baixo quando comparado às observações de WHEELER \& THRELFALL (1986), ao registrarem valores de 41,0\%, apenas em aves passeriformes. considerando apenas este grupo de aves, o percentual ora encontrado ficaria ainda menor (6,87\%). CLAYTON et al. (1992) assinalaram 47,7\% e BOYD (1951) quando estudou o ectoparasitismo em Sturnus vulgaris (Sturnidae), indicam 93,4\%. Recentemente, MARINI et al. (1996) constataram uma prevalência de infestação por phthirápteros de $66,7 \%$ em aves da Floresta Atlântica do sul do Brasil e, MARINI \& COUTO (1997), 19,0\% para aves de cerrado e matas de Minas Gerais.

Ainda que a prevalência seja função de vários fatores, tais como dieta, idade e habitat do hospedeiro, CLA YTON et al. (1992) ao compararem seus resultados com os de WheEler \& Threlfall (1986), constataram que os phthirápteros de aves tropicais, não seriam mais abundantes que aquelas das regiões temperadas, considerando-se que os phthirápteros parasitos de aves sejam mais dependentes das condições do corpo do hospedeiro do que propriamente das condições ambientais.

AGRADECIMENTOS. Agradecemos à CAPES pela bolsa de mestrado. Ao Curso de Mestrado em Biologia Animal da Universidade Federal de Pernambuco; ao auxílio em campo de Gilmar Farias, Fabrícia Leal, Roberta Rodrigues e Rossano Mendes Pontes; ao Prof. Galileu Coelho (UFPE) pela co-orientação deste trabalho e à Dra. Yoshika Oniki (UNESP) pela identificação dos phthirápteros.

Revta bras. Zool. 16 (3): 871 - 878, 1999 


\section{REFERÊNCIAS BIBLIOGRÁFICAS}

AsH, J.S. 1960. A study of the Mallophaga of birds with particular reference to their ecology. Ibis 102: 93-110.

ASKEW, R.R. 1973. Parasitic Insects. London, Heinemann Educational Books, $316 p$.

BAER, J.G. 1971. EI Parasitismo Animal. Madrid, Ediciones Guadarrama, 256p.

BARKER, S.C. 1994. Phylogeny and classification, origins, and evolution of host associations of lice. Int. Jour. Parasit. 24 (8): 1285-1291.

BARKER, S.C.; D.A. Briscoe; R.L. Closeo \& P. DALlas. 1991. Genetic variation in the Heterodoxus octoseriatus group (Phthiraptera): a test of Prince's model of parasite evolution. Int. Jour. Parasit. 21 (5): 555-563.

BoYD, E.M. 1951. The external parasites of birds: a review. Wilson Bull. 63 (4): 363-369.

CARRIKER JR., M.A. 1960. Studies in Neotropical Mallophaga, XVII: a new family (Trochiliphagidae) and a new genus of the lice of hummingbirds. Proc. U.S. Nat. Mus. 112: 307-342.

CHANDLER, A.C. 1955. Introduction to Parasitology. With special reference to the parasites of the man. New York, Jonh Wiley \& Sons, 799p.

ChEng, T.C. 1964. The Biology of Animal Parasites. Philadelphia, W.B. Sauders Company, $727 \mathrm{p}$.

CLAY, T. 1949. Piercing mouth-parts in the biting lice (Mallophaga). Nature 4171: 617.

Clayton, D.H.; R.D. Gregory \& R.D. Price. 1992. Comparative ecology of neotropical lice (Insecta: Phthiraptera). Jour. Anim. Ecol. 61: 781-795.

FigueIredo, S.M.; J.H. GUIMARÃES \& N.M.S.Q. GAMA. 1993. Biologia e ecologia de malófagos (Insecta, Phthiraptera) em aves de postura de granjas industriais. Rev. Bras. Parasitol. Vet. 2 (1): 45-51.

FosTER, M. 1969. Synchronized life cycle in the orange-crowned warbler and its mallophagan parasites. Ecology 50 (2): 315-123.

KARTMAN, L. 1950. On the host-ectoparasite relation. Jour. Econ. Entom. 43 (3): 385-386.

MARGOlis, L.; G.W. EsCH; J.C. Holmes; A.M. KuRIS \& G.A. SCHAD. 1982. The use of ecological terms in parasitology (Reported of an ad hoc Committee of the American Society of Parasitologists). Jour. Parasit. 68 (1): 131-133.

MARINI, M.A.; B.L. REINERT; M.R. BORNSCHEIN; J.C. PINTO \& M.A. PICHORIM. 1996. Ecological correlates of ectoparasitism on Atlantic Forest birds, Brazil. Ararajuba 4 (2): 93-102.

MARINI, M.A. \& D. COUTO. 1997. Correlações Ecológicas entre ectoparasitas e aves de florestas de Minas Gerais, p. 210-218. In: L.L. LeITE \& C.H. SAITO (Eds). Contribuição ao Conhecimento Ecológico do Cerrado. - Trabalhos selecionados do $3^{\circ}$ Congresso de Ecologia do Brasil. Brasília, Departamento de Ecologia, Universidade de Brasília.

MARSHALL, A.G. 1981. The Ecology of Ectoparasitic Insects. New York, Academic Press, 459p.

Noble, E.R. \& G.A. Noble. 1965. Parasitologia. Biología de los Parásitos Animales. Ciudad de Mexico, Interamericana, 675p. 
ONIKI, Y. 1990. Survey of lice (Mallophaga) and some remarks on their life cicle on birds at Balbina, Amazonas, Brazil. Rev. Brasil. Biol. 50 (3): 615-617.

. 1995. Trochiliphagus austini sp.n. (Mallophaga, Trochiloecetidae) from Amazilia candida (Aves, Trochilidae) from Belize, with biological notes. Iheringia, sér. Zool., 78: 67-71.

ONIKI, Y. \& K.C. EMERSON. 1982. A new species of Trochiloecetes (Mallophaga: Ricinidae) from the saw-billed hermit, Ramphodon naevius (Dumont) (Apodiformes: Trochilidae). Rev. Brasil. Biol. 42 (1): 85-87.

ONIKI, Y. \& E.O. WILLIS. 1991. Morphometrics, molt, cloacal temperatures and ectoparasites in Colombian birds. Caldásia 16 (79): 519-524.

RòzSA, L. 1993. Speciations patterns of ectoparasites and "straggling" lice. Int. Jour. Parasit. 23 (7): 859-864.

Stock, T.M. \& L.E. HunT. 1989. Site specificity of three species of lice, Mallophaga, on the willow ptarmigan, Lagopus lagopus, from Chilkat Pass, British Columbia (Canada). Can. Field Nat. 103 (4): 584-588.

Stenram, H. 1956. The ecology of Columbicola columbae L. (Mallophaga). Opusc. Ent. 21 (2-3): 170-190.

WHEELER, T.A. \& W. THRELFALL. 1986. Observations on the ectoparasites of some Newfoundland passerines (Aves: Passeriformes). Can. Jour. Zool. 64: 630-636.

Recebido em 27.II.1998; aceito em 27.VIII.1999. 